\title{
The actin gene of the glaucocystophyte Cyanophora paradoxa: analysis of the coding region and introns, and an actin phylogeny of eukaryotes
}

Received: 4 December 1996 / Accepted: 5 February 1997

\begin{abstract}
We isolated the actin gene of the glaucocystophyte alga Cyanophora paradoxa and analyzed the coding region and its introns. Phylogenetic analyses of the actin coding region and the inferred protein sequence in data sets containing 47 other actin sequences show Cyanophora to be a member of the eukaryotic crown-group radiation in agreement with ribosomal DNA sequence analyses. Four of the five Cyanophora actin introns are relatively short (55-59 nt) and occupy novel positions in a catalogue of actin introns containing 56 distinct sites. The fifth intron has a length of $171 \mathrm{nt}$ and occurs also in actin genes from green algae and the crustacean Artemia.
\end{abstract}

Key words Actin - Cyanophora paradoxa - Introns · Phylogeny

\section{Introduction}

The glaucocystophytes represent a small (nine genera, Kies 1979; Kies and Kremer 1990), morphologically distinct, protist group that is distinguished by its photosynthetic organelles, the cyanelles. Characters such as cruciate flagellar roots with associated multilayered structures (MLS) have suggested a phylogenetic relationship between glaucocystophytes and the green algae (Melkonian 1983), whereas cyanelle characters have been used to position glaucocystophytes with the red algae (Cavalier-Smith 1982). Cyanelles resemble red-algal plastids in that they contain only chlorophyll- $a$ and phycobilisomes and have unstacked thylakoids (Kies and Kremer 1990). Cyanelles were previously thought to represent recent, possibly independent, endosymbioses because these organelles main-

D. Bhattacharya $(\bowtie) \cdot K$. Weber

Department of Biochemistry,

Max Planck Institute for Biophysical Chemistry,

Am Fassberg 11, D-37077 Göttingen, Germany

Communicated by R. Schweyen tain the peptidoglycan wall of the cyanobacterium as a bounding plastid membrane. Phylogenetic analyses of nuclear-encoded small subunit ribosomal DNAs (SSU rDNAs) identify the glaucocystophytes as a member of the eukaryotic "crown-group" radiation and a sister group to the cryptophyte algae (with weak bootstrap support, see Bhattacharya and Medlin 1995). Glaucocystophytes do not share a specific relationship with red or green algae in rDNA phylogenies (Bhattacharya et al. 1995). Analyses of the cyanelle-encoded rDNAs show these organelles to be "advanced" plastids that form a monophyletic group closely related to red- and green-algal plastids (Helmchen et al. 1995).

To gain another perspective on the phylogeny of the glaucocystophytes, we have determined the sequence of the single-copy actin gene of Cyanophora paradoxa and included it in phylogenies based on actin sequences from primitive eukaryotes as well as members of the crown groups. Actin is a highly conserved eukaryotic protein that can be used in phylogenetic analyses to corroborate results based on rDNA and other protein-sequence analyses (Bhattacharya et al. 1991, 1993; Baldauf and Palmer 1993; Bouget et al. 1995; Drouin et al. 1995). Previous actin trees have, for example, been consistent with a sister-group relationship between animals and fungi proposed on the basis of rDNA sequence analyses (Baldauf and Palmer 1993; Wainright et al. 1993), and an early divergence of the archezoan Giardia l. from the eukaryote phylogeny (Sogin et al. 1989; Baldauf and Palmer 1993; Drouin et al. 1995). Actin trees have also sometimes conflicted with the results of rDNA sequence comparisons (e.g. the position of slime molds, see Bhattacharya et al. 1991; Drouin et al. 1995 and below) and provided the impetus to probe more deeply into the resolving power of rDNA and protein-sequence phylogenies (Loomis and Smith 1990). For these reasons, actin-sequence comparisons are an important addition to analyses of eukaryote/gene phylogeny, and in those cases where actin exists as a single-copy coding region (e.g. fungi, ciliates, most green algae) it is taken as a working hypothesis that these sequences may be used as a molecular marker. In other lineages where actin genes form multi- 
copy families (e.g. land plants, metazoa) these sequences can be used to study aspects of concerted gene evolution and, within orthologous subfamily members, may also be used as phylogenetic markers (Moniz de Sá and Drouin 1996).

The analyses of spliceosomal intron positions is also well established with actin genes due to the highly conserved sequence and length of most of this protein (except for the variable $\mathrm{N}$-terminus) that allow for the unambiguous alignment of intron sites. The existence of a putative proto-splice site bordering intron insertion positions [i.e. $(\mathrm{C} / \mathrm{A}) \mathrm{AG}$-intron- $(\mathrm{A} / \mathrm{G})]$ was, for example, recognized on the basis of actin and tubulin gene analyses (Dibb and Newman 1989). In the present paper we have incorporated the Cyanophora actin introns into an updated catalogue of actin spliceosomal intron positions among all eukaryotes (see also Weber and Kabsch 1994).

\section{Materials and methods}

Isolation of the actin DNA clone. Genomic DNA was isolated from an actively growing culture of C. paradoxa Korsch. [Kies strain, Sammlung von Algenkulturen Göttingen (Schlösser 1984), SAG B 45.84] as previously described (Helmchen et al. 1995). A genomic DNA bank was established from this taxon using the BamHI-digested, de-phosphorylated vector Lambda-Dash (Stratagene) and partially digested BamHI genomic DNA from Cyanophora. Ligation and packaging of the vector and DNA were done according to the manufacturer's instructions (Stratagene). The bank was amplified (50 000 original PFU) and stored in SM buffer $[10 \mathrm{mM} \mathrm{NaCl}, 10 \mathrm{mM}$ $\mathrm{MgCl}_{2}, 50 \mathrm{mM}$ Tris- $\mathrm{HCl}$ ( $\left.\mathrm{pH} 7.5\right), 2 \%$ w/v gelatin] with $5 \%$ chloroform. The genomic bank of Cyanophora was screened with an actin-encoding cDNA PCR fragment isolated from this taxon in $6 \times \mathrm{SSC}, 30 \%$ formamide and $5 \times$ Denhardt's solution at $40^{\circ} \mathrm{C}$, overnight. The actin probe was isolated with a RT/PCR method using the forward primer 244F (5'-GAYATGGAAAAGATCTGG-3') and a universally conserved reverse primer at the carboxy terminus of actin-coding regions (DBAc3, 5'-CCGAATTCTGCAGARAAGCAYTTS CTGTG-3'; see Bhattacharya et al. 1991, 1993). Filters were washed twice in $2 \times \mathrm{SSC}, 0.1 \% \mathrm{SDS}$ at $40^{\circ} \mathrm{C}$. Actin-containing phages were isolated and a XbaI fragment (of size $2.2 \mathrm{~kb}$ ) from one of these phages, that cross-reacted with the Cyanophora RT/PCR actin fragment in a Southern analysis, was subcloned into the $X b a \mathrm{I}$ restriction site in pBluescript for sequencing. Actin sequences were determined over both strands of this gene using primers complementary to conserved regions within all actin-coding regions (Bhattacharya et al. 1991), or by primer-walking using Cyanophora-specific actin oligonucleotides and the dideoxy sequencing protocols (Sanger et al. 1977) with a T7 sequencing kit (Pharmacia). The XbaI fragment characterized encodes the actin genomic sequence lacking approximately $57 \mathrm{nt}$ at the $3^{\prime}$ end of the coding region. The remaining genomic actin sequence of Cyanophora could not be found within the original Lambda-Dash clone. To extend the 3'-terminus of the actin-coding region, the RT/PCR method was used with Cyanopho$r a$ total RNA extracted with an RNeasy kit (Qiagen). First-strand synthesis was initiated with an oligo-dT 12-18 $_{\text {primer (Pharmacia). }}$ The actin-coding region was amplified from this cDNA using the forward primer 613F (TTCACCACCACTGCTGAG-3') and the reverse primer, DBAc3. This PCR fragment was cloned into the SmaI restriction site in pBluescript and sequenced using the T3 and T7 primers. The actin gene sequence of Cyanophora is available from the EMBL/GenBank/DDBJ databases under the accession number, U90325.

Southern analysis. Genomic DNA was prepared from Cyanophora as described above and digested to completion with the restriction enzymes SalI, SacI and SmaI. The restriction products were sizefractionated in a $1 \%$ agarose gel in $1 \times \mathrm{TBE}(89 \mathrm{mM}$ Tris $\mathrm{Cl}, 89 \mathrm{mM}$ boric acid, $2 \mathrm{mM}$ EDTA) electrophoresis buffer and transferred onto a nitrocellulose membrane (Schleicher and Schuell) and baked at $80^{\circ} \mathrm{C}$ for $2 \mathrm{~h}$. The Southern blot was pre-hybridized at $40^{\circ} \mathrm{C}$ in a $6 \times$ SSC, $50 \%$ formamide, $5 \times$ Denhardt's and $0.1 \%$ SDS solution for $2 \mathrm{~h}$ and then incubated overnight in $5 \times \mathrm{SSC}, 50 \%$ formamide, $1 \times$ Denhardt's and $0.1 \%$ SDS with the $613 \mathrm{~F}-\mathrm{DBAc} 3$ Cyanophora actin RT/PCR fragment as probe. The actin fragment was radioactively-labelled with ${ }^{32} \mathrm{P}$-dCTP using a random-primed oligolabelling kit (Pharmacia). The Southern blot was washed twice for $1 \mathrm{~h}$ each time in $2 \times \mathrm{SSC}, 0.1 \% \mathrm{SDS}$ at $50^{\circ} \mathrm{C}$ prior to autoradiography. A further more-stringent wash was done in $0.1 \times \mathrm{SSC}, 0.1 \% \mathrm{SDS}$ at $55^{\circ} \mathrm{C}$ for $1 \mathrm{~h}$ with the same membrane to test the specificity of the actinannealing fragments resolved in the first autoradiogram.

Phylogenetic analyses. The actin-coding region from Cyanophora was aligned with homologous sequences from 47 other eukaryotes and two centractin sequences (actin-related proteins; see Clark and Meyer 1992; Lees-Miller et al. 1992; Sheterline et al. 1995) from man and dog. Two data sets were prepared from this alignment. The first data set included the centractin sequences as the outgroup for eukaryotes ranging from Giardia to members of the crown group. This data set was used to determine the order of divergence of the most primitive eukaryotes in the analyses (e.g. Giardia, Trypanoso$m a b$.) and their phylogenetic position relative to the crown-group radiation. The gene duplication that resulted in the actin and centractin genes is presumed to have occurred in the common ancestor of all eukaryotes (Bhattacharya and Ehlting 1995; Sheterline et al. 1995). The second data set included a larger number of actin sequences from crown-group eukaryotes than in the first data set, with the apicomplexan (Plasmodiumf., Toxosplasma g.) actin-coding regions as an outgroup. The apicomplexans diverge prior to the crown-group radiation in actin phylogenies (see also Baldauf and Palmer 1993; Drouin et al. 1995). The second data set was used to infer the phylogenetic relationships of the crown-group lineages. The first and second nucleotide positions of actin codons (748 nt total) in both data sets were submitted to a weighted [re-scaled consistency (RC) index over the interval 1-1000] maximum-parsimony (MP) analysis (PAUP V3.1.1, Swofford 1993) using a heuristic search procedure with a branch-swapping algorithm (TBR, tree bisection-reconnection). The stability of internal nodes within the MP actin phylogenies was studied with a bootstrap analysis [ 100 replications (first data set), 200 replications (second data set), Felsenstein 1985]. Both data sets were also analyzed with a distance method using a Kimura (1980) matrix as input for a neighbor-joining phylogenetic reconstruction (Saitou and Nei 1987) with jumbled taxon addition and the transition/transversion ratio $=2$ (Phylip V.3.5c, Felsenstein 1993). Bootstrap analyses (200 replications, Felsenstein 1985) were done with the distance method. Further, the phylogeny of the actin protein sequences in the second data set (372 amino acids) was studied using a distance method (PROTDIST, Dayhoff distance matrix, Phylip V.3.5c) and a neighbor-joining phylogenetic reconstruction. Onehundred bootstrap re-samplings were used to test the stability of the internal nodes in the protein distance tree. All actin sequences used in these analyses are available from the EMBL/GenBank/DDBJ databases.

\section{Results and discussion}

Southern analysis of the Cyanophora actin-coding region

Southern analysis using the actin RT/PCR probe shows one actin-annealing band of approximate size $1100 \mathrm{nt}$ in the SalI restriction digest, and two bands of approximate sizes $1050 \mathrm{nt}$ and $3000 \mathrm{nt}$ in the PstI digest of Cyanophora genomic DNA (Fig. 1). Restriction analysis of the XbaI fragment encoding the genomic Cyanophora actin sequence 


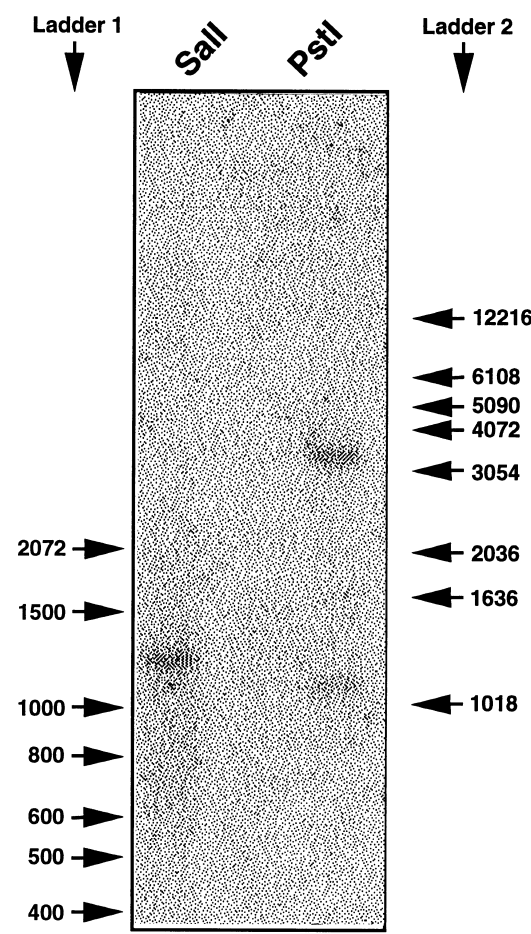

Fig. 1 Southern analysis of the Cyanophora genomic DNA with a homologous actin-cDNA probe. Ladders $1(100 \mathrm{bp})$ and $2(1 \mathrm{~kb})$ are from BRL shows an internal PstI restriction site $300 \mathrm{nt}$ from the 3' terminus of this insert. A single band of approximate size $2.1 \mathrm{~kb}$ was resolved when Cyanophora genomic DNA digested with SmaI was included in the Southern analysis (data not shown). These results were also found after more stringent washing of the membrane (see Materials and methods). Sequence analysis (T3, T7 primers) of five independent clones isolated from the RT/PCR experiments (613F+DBAc3 PCR primers) with Cyanophora also showed identity indicating that there is one actin-encoding mRNA expressed in Cyanophora. The PCR primers are not likely to have bound to only one class of actin mRNAs since these oligonucleotides recognize highly conserved regions of the coding region. The results of the Southern and RT/PCR-sequence analyses are consistent with the existence of a single-copy actin gene in the genome of Cyanophora.

\section{Evolutionary analysis of the Cyanophora actin gene}

The actin gene of Cyanophora is interrupted by five spliceosomal introns that occur at positions 44-1, 248-3, 283-2, 318-3 and 345-2 relative to the vertebrate skeletal muscle $\alpha$-actin sequence (Fig. 2). To determine if additional introns may be located in the 3 -terminal sequence that was determined from the cDNA, a PCR amplification
Fig. 2 The Cyanophora actin sequence determined from a genomic clone and the actin cDNA (see text for details). The coding region is shown in upper case whereas the five introns are shown in lower case. This sequence begins with the putative methionine start codon in actin and ends with the sequence of the PCR primer DBAc3 (shown in bold letters) used to isolate the cDNA. Nucleotides that could not be unambiguously determined are indicated with the letter $N$. The putative branch-point sequences within the introns are underlined and the consensus branchpoint sequence with the branch point adenine in shadowed print is shown at the bottom of the figure
1 ATGGAAGACGAGGTTGCGGCGCTGGTCATTGACAATGGCACCGGCATGTGCAAGGCCGGCTTCGCCGGCGATGCCGCCCGCGTGCCGTGTTCCCCGTCA M--E--D--E--V--A--A--L--V--I--D--N--G--T--G--M--C--K--A--G--F--X--X--D--A--A--R--R--A--V--F--P--V--

101 TCGTGGGTGCCCCGCGCCACCAAGgtccgttgttctggaggctccgcattccgtgtgtccaaaactcgaaaaccgcgctcaccgcctaataaaaccgtta I--V--G--A--P--R--H--Q--

201 cgctagacaagttatacgtattagaggccttctaacgcccggccgcttcgttcggttgcattttacttacattcaaaaatggttgttctcggcagGCGTC

301 ATGGTCGGCATGGGGCAGAAGGACTCGTATGTCGGCGACGAGGCGCAGTCGAAGCGTGGTATCCTCACGCTGAAGTACCCGATTGAGCACGGCATCGTGA -M--V--G--M--G--Q--K--D--S--Y--V--G--D--E--A--Q--S--K--R--G--I--L--T--L--K--Y--P--I--E--H--G--I--V--

401 CCAACTGGGACGACATGGAGAAGATTTGGCACCACACCTTCTACAACGAGCTCCGCGTTGCCCCCGAGGAGCACCCCGTTCTGCTCACTGAGGCGCCGCT T--N--W--D--D--M--E--K--I--W--H--H--T--F--Y--N--E--L--R--V--A--P--E--E--H--P--V--L--L--T--E--A--P--L

501 GAACCCGAAGGCAACCGCGAGAAGATGACCCAGATCATGTTCGAGACCTTCAACGTGCCGGCGATGTACGTTGCCATCCAGGCCGTGCTCTCCCTCTAC --N--P--K--A--N--R--E--K--M--T--Q--I--M--F--E--T--F--N--V--P--A--M--Y--V--A--I--Q--A--V--L--S--L--Y-

601 GCCTCCGGCCTACCACCGGTATCGTGCTCGACTCCGGCGATGGTGTCACCCACACCGTGCCCATCTACGAGGGTTANGCGCTCCCGCACGCCATCCTCC -A--S--G--R--T--T--G--I--V--L--D--S--G--D--G--V--T--H--T--V--P--I--Y--E--G--X--A--L--P--H--A--I--L--

701 GTCTCGACCTCGCCGGCCGCGACCTCACCGACTACCTCATGAAGATCCTCACCGAGCGCGGCTACTCCTTCACCACCACCGCTGAGCGTGAAATCGTCCG R--L--D--L--A--G--R--D--L--T--D--Y--L--M--K--I--L--T--E--R--G--Y--S--F--T--T--T--A--E--R--E--I--V--R

801 CGACATCAAGGAGAAGCTCTGCTACGTCGCCCTGGACTTCGAGACCGAGATGCAGACCGCCTCGACCTCCAGCACCGTCGAGAAGACCTACGAGCTTCCG --D--I--K--E--K--L--C--Y--V--A--L--D--F--E--T--E--M--Q--T--A--S--T--S--S--T--V--E--K--T--Y--E--L--P-

901 GACGGCCAGgtcagtttagatataaaatgttgttctccttctctttttqaccccgggcgcccccgcagGTCATCACCATCGGCAACGAGGGCTTCCGGTG

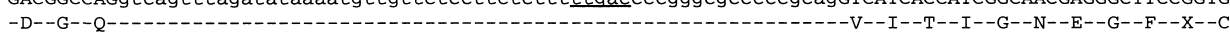
1001 CCCGGAGGTCCTCTTCCAGCCCAGCTTCATCGGCATGGAGTCGGCCGGCATCCACGAGACCACCTACAACAGgtccgcgtt tcctt tgtgtcgccctccC --P--E--V--L--F--Q--P--S--F--I--G--M--E--S--A--G--I--H--E--T--T--Y--N--R--10 1101 tccgcttcgagctgatccgttcccgcagCATCATGAAGTGCGACGTCGATATTCGTAAGGACCTGTACGGCAACATCGTGCTCTCCGGCGGCACCACCAT 1201 GTACGCTGGCATCNGNGACCGCATGCAGAAGGAGgtccgtagttatctggtttcggctcctggt tt tatgatt taaccCtt tgcCCagATCACCGCCCT --Y--A--G--I--X--D--R--M--Q--K--E--

1301 GGCGCCCTCGTCGATGAAGATCAAGATCGTCGCGCCCCCGAGCGCAAGTACTCTGTCTGGATCGGCGGgttcgcccttttctcgcttatgtggtccaa --A--P--S--S--M--K--I--K--I--V--A--P--P--E--R--K--Y--S--V--W--I--G--G--

1401 tcagcccactgaagccgttgccctcagGTCTATCCTTGCCTCGCTCTCCACCTTCCAGCAGATGTGGATCTCGAAGCAGGAGTACGACGAGTCCGGCCCC -

1501 TCGATCGTCCACAgGaAatgctTt * $-S--T--V--H--R--K--C--F$ 


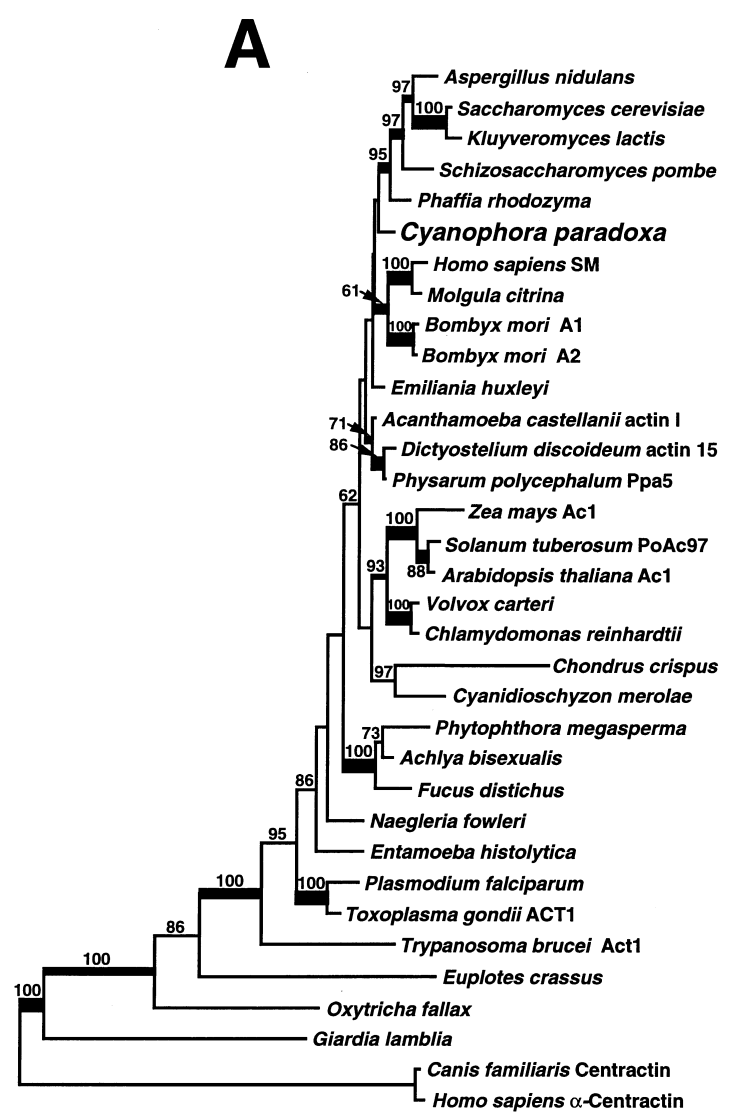

Fig. 3 A, B Phylogeny of actin-coding regions. A tree inferred with a weighted (RC index over the interval 1-1000) MP method (PAUP V3.1.1, Swofford 1993) using a heuristic search procedure with a branch-swapping algorithm (TBR, tree bisection-re-connection) and the first and second positions of actin codons (748 nucleotides). The bootstrap (100 replications)-consensus phylogeny shown has a consistency index of 0.620 . The bootstrap values are presented at the internal nodes. This tree has been rooted within the branch-length leading to the centractin sequences. B phylogenetic analysis of actin coding regions in crown group eukaryotes done as described above. The bootstrap (200 replications)-consensus phylogeny shown has a consistency index of 0.686 . The bootstrap values presented above the internal nodes were inferred from the MP analyses, whereas the values shown below the nodes were inferred from a bootstrap analysis (100 replications) using the actin protein sequences and the PROTDIST method (Phylip V.3.5c, Felsenstein 1993) with a neighbor-joining tree re-construction. This tree has been rooted within the branch length leading to the apicomplexan actin sequences. The results of bootstrapped distance analyses (200 replications) of both data sets using the first and second positions of the actins codons with a Kimura (1980) model and a neighbor-joining tree reconstruction are shown as branch lengths of differing thicknesses. The legend to these bootstrap values is shown next to $\mathbf{B}$. The asterisk in $\mathbf{B}$ marks the putative origin of a homologous family of introns found in the actin genes of the ascomycetous fungi to the right of this symbol (see text for details). Only bootstrap values $\geq 60 \%$ are shown and are indicated with arrows where they do not fit on the branches

was done using the primers $613 \mathrm{~F}$ and $\mathrm{DBAc} 3$ with genomic DNA from Cyanophora. A fragment of approximate size $760 \mathrm{nt}$ was isolated and verified as encoding actin using a Southern analysis with the Cyanophora cDNA fragment as a probe. These data suggest that no more introns inter-

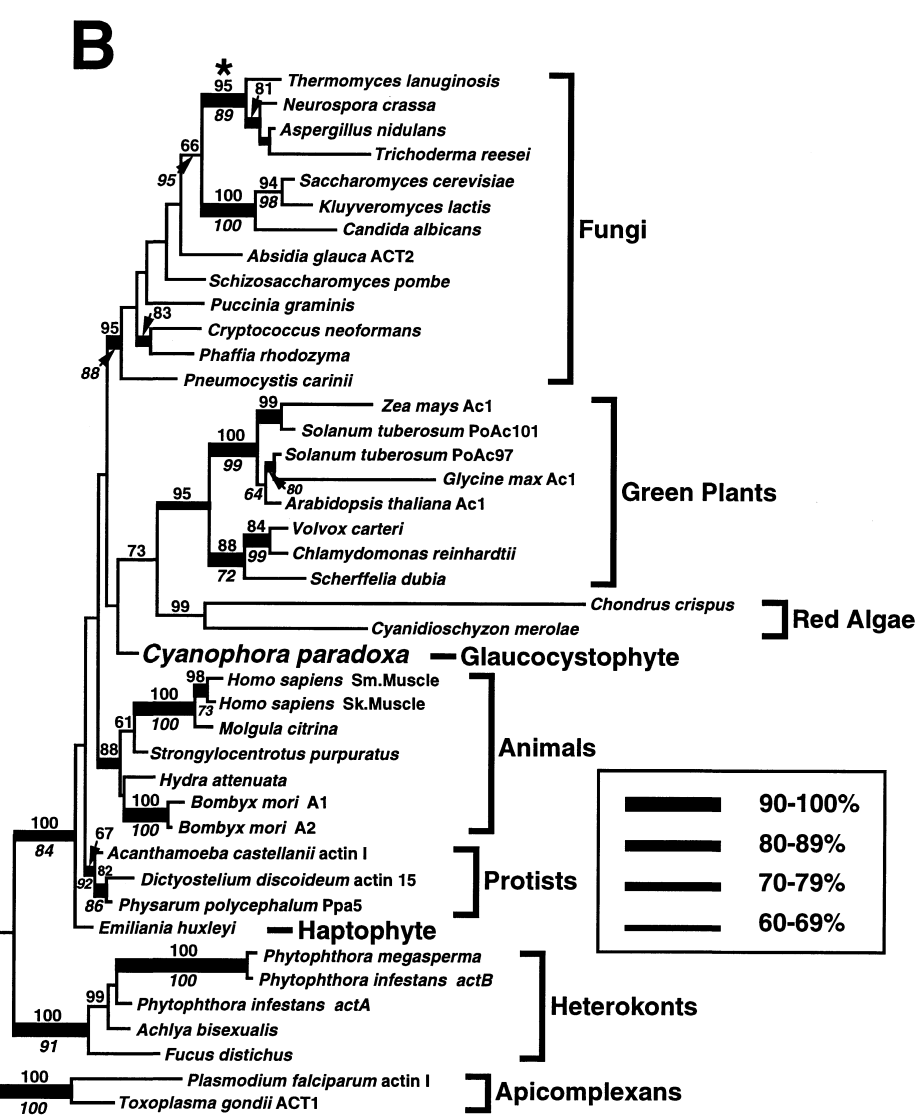

rupt the 3 -terminal end since the expected size of the genomic actin sequence with the 3 '-terminal sequence determined from the cDNA is also $760 \mathrm{nt}$. The Cyanophora actin introns all have the consensus $\mathrm{GT}^{\mathrm{C}} / \mathrm{T} / \mathrm{A}$ with $\mathrm{CAG}$ donor and acceptor sequences at the $5^{\prime}$ and $3^{\prime}$ termini of the introns, respectively (Fig. 2 B, Jackson 1991). A putative branch-point sequence was identified in each Cyanophora actin intron (underlined in Fig. 2). The consensus of these branch-point sequences (shown below Fig. 2) maintains an adenosine at the branch site that has been shown to be crucial for the chemical steps of splicing (Query et al. 1996). The Cyanophora consensus branch-point sequence is similar to that in higher plants (i.e. $\mathrm{CT}^{\mathrm{A}} / \mathrm{G}^{\mathrm{C}} / \mathrm{T}$, Sinibaldi and Mettler 1992).

The Cyanophora coding region was readily aligned with other actin sequences and the phylogenetic analyses of the DNA and protein sequences position this alga as an independent lineage within the crown-group radiation (Fig. 3 A, B). Actin sequences are needed from cryptophyte algae to test the sister-group relationship between the glaucocystophytes and cryptophytes identified in the rDNA phylogenies (Bhattacharya and Medlin 1995). Inclusion of the centractin sequences as an outgroup identifies the archezoan Giardia as the earliest divergence within the eukaryote actin phylogeny (Fig. $3 \mathrm{~A}$, see also Baldauf and Palmer 1993; Drouin et al. 1995). Many of the major crown-group lineages (e.g. fungi, animals, green plants, heterokonts) form monophyletic groups with bootstrap support in the actin phylogenies (see in particular Fig. 3 B). 
The ciliate actin sequences (i.e. Euplotes $c$., Oxytricha f.) do not however group either with themselves or with the apicomplexans, as would be expected on the basis of rDNA and morphological analyses (Cavalier-Smith 1993). This result may stem from the high divergence of the ciliate actin-coding regions and their resultant homoplasious "attraction" (Swofford and Olsen 1990) to the outgroup taxa (see also Drouin et al. 1995).

The fungi and animals which form a lineage with no bootstrap support in Fig. $3 \mathrm{~A}$ do not share a common origin in the the phylogeny shown in Fig. 3B or in the protein distance tree (data not shown). This result is at odds with comparisons of rDNA (Wainright et al. 1993) and protein-coding (e.g., $\alpha$-, $\beta$-tubulin, EF- $1 \alpha$; Baldauf and Palmer 1993) sequences and previously published actin phylogenies (Bhattacharya and Ehlting 1995; Drouin et al. 1995). A likely explanation for this inconsistency is the inclusion of actin sequences from protists that are either single representatives of their phylum or have very low sequence-divergence rates (e.g. slime molds) in the phylogenetic analyses (see Baldauf and Palmer 1993 for discussion). Exclusion of the Acanthamoeba c., Dictyostelium d., Physarum p., Emiliania $h$. and Cyanophora actin sequences from the data set shown in Fig. $3 \mathrm{~B}$ resulted, for example, in bootstrap support for the monophyly of the fungi and animals. The distance analysis of nucleotides encoding the first and second positions of actin codons resulted in a bootstrap value of $85 \%$ (200 replications), while the MP analysis of the same data set resulted in a bootstrap value of $83 \%$ (100 replications) in support of the animalfungal clade; the distance tree also grouped the green plants and red algae together as in the weighted MP analysis (Fig. 3 B) though with no bootstrap support (56\%). Analysis of the reduced data set of protein sequences resulted in a bootstrap value of $80 \%$ in support of the animal-fungal clade.

An additional reason for the weak bootstrap support for some well-established monophyletic groups in the actin phylogenies (e.g. animals in the MP analysis shown in Fig. $3 \mathrm{~A}, 61 \%$ ) is most likely due to variation in sequencedivergence rates. A high sequence-divergence-rate variation within and between lineages (Bhattacharya et al. 1991) may be the most important problem that hinders the use of actin and other molecular markers as gene/species markers (see also Palmer and Delwiche 1996). It has been shown, for example, with the relative-rate test that Dictyostelium and Physarum, which unexpectedly form a cluster within the crown-group in actin phylogenies (Fig. 3 B, Bhattacharya et al. 1991, 1993; Drouin et al. 1995), have a significant sequence-divergence-rate slowdown in comparison to other crown-group members (e.g. green plants, Drouin et al. 1995). Dictyostelium and Physarum diverge well before the crown-group radiation in rDNA sequence analyses (Sogin 1991; Cavalier-Smith 1993; McFadden et al. 1994).

In spite of these difficulties the use of protein codingsequence phylogenies is an important component of molecular evolutionary studies. One result, for example, that was found in the MP analyses of both data sets, as well as in the distance analyses of the second data set with and without Acanthamoeba, Dictyostelium, Physarum, Emiliania and the Cyanophora actin sequences, appears to be promising and deserves further study. These trees are consistent with a sister-group relationship between green plants and red algae and therefore with a monophyletic origin of the plastid in these groups. In plastid SSU rDNA and protein (except $r b c \mathrm{~S}, \mathrm{~L}$ ) phylogenies, the red- and green-algal plastids form monophyletic lineages that are sister groups of each other with a common root within the cyanobacteria. The glaucocystophyte cyanelles form a third plastid lineage that is also closely related to red- and green-algal plastids (Morden et al. 1992; Helmchen et al. 1995). The actin phylogenies suggest that the red- and green-algae may have had a single origin of their plastids in their common ancestor. The nuclear-encoded rDNA sequence comparisons have not yet been able to identify conclusively the sister group of green plants and red algae and have therefore not been useful for tracing the origin(s) of their plastids (Bhattacharya and Medlin 1995). As more actin sequences become available from these groups, it will be interesting to see if the glaucocystophytes also group with bootstrap support with the red algae and green plants (as in Fig. 3 B).

\section{Actin intron catalogue}

Four of the five Cyanophora actin introns (44-1, 283-2, $318-3,345-2)$ occupy novel sites in the catalogue of actin intron positions, whereas the fifth intron (248-3) is also found in the actin gene of the green algae Volvox $c$. and Chlamydomonas $r$. and in two genes of the crustacean Artemia (Fig. 4). The sizeable increase in the total number of actin intron positions from 40 (Weber and Kabsch 1994; Sheterline et al. 1995) to 56 (Fig. 4) is due to the addition of four Cyanophora introns (see above), one Artemia intron (303-1), and the 11 fungal introns (3-2, 7-1, 10-3, 232, 34-2, 57-2, 121-1, 256-2, 312-1, 339-2, 359-1) found in Phaffia r., Cryptococcus $n$. and Puccinia g. On the proteinsequence level, 2 of these 16 new intron positions (34-2 and 44-1) are not resolved from two previously documented introns (34-1 and 44-2) and one position (3-2) lies in the variable N-terminal region. Thus 13 new intron positions can be analyzed.

A previous analysis of 36 intron positions on the protein-sequence level showed that 23 positions are found at the ends of secondary structural elements ( $\beta$ strand, $\alpha$-helix and $3 / 10$ helix) plus or minus one residue. Thirteen positions do not show this relationship and eight interrupt a secondary structural element. Statistical analysis showed that, due to the large number of end positions in the actin molecule, the boundaries of secondary structural elements are not correlated with intron positions (for details see Weber and Kabsch 1994). Using the same approach for the 13 novel intron positions reported here, eight are found at the ends of secondary structural elements plus or minus one residue (10-3, 23-2, 57-2, 256-2, 303-1, 312-1, 339-2 and 359-1) whereas five are found elsewhere (7-1, 121-1, 


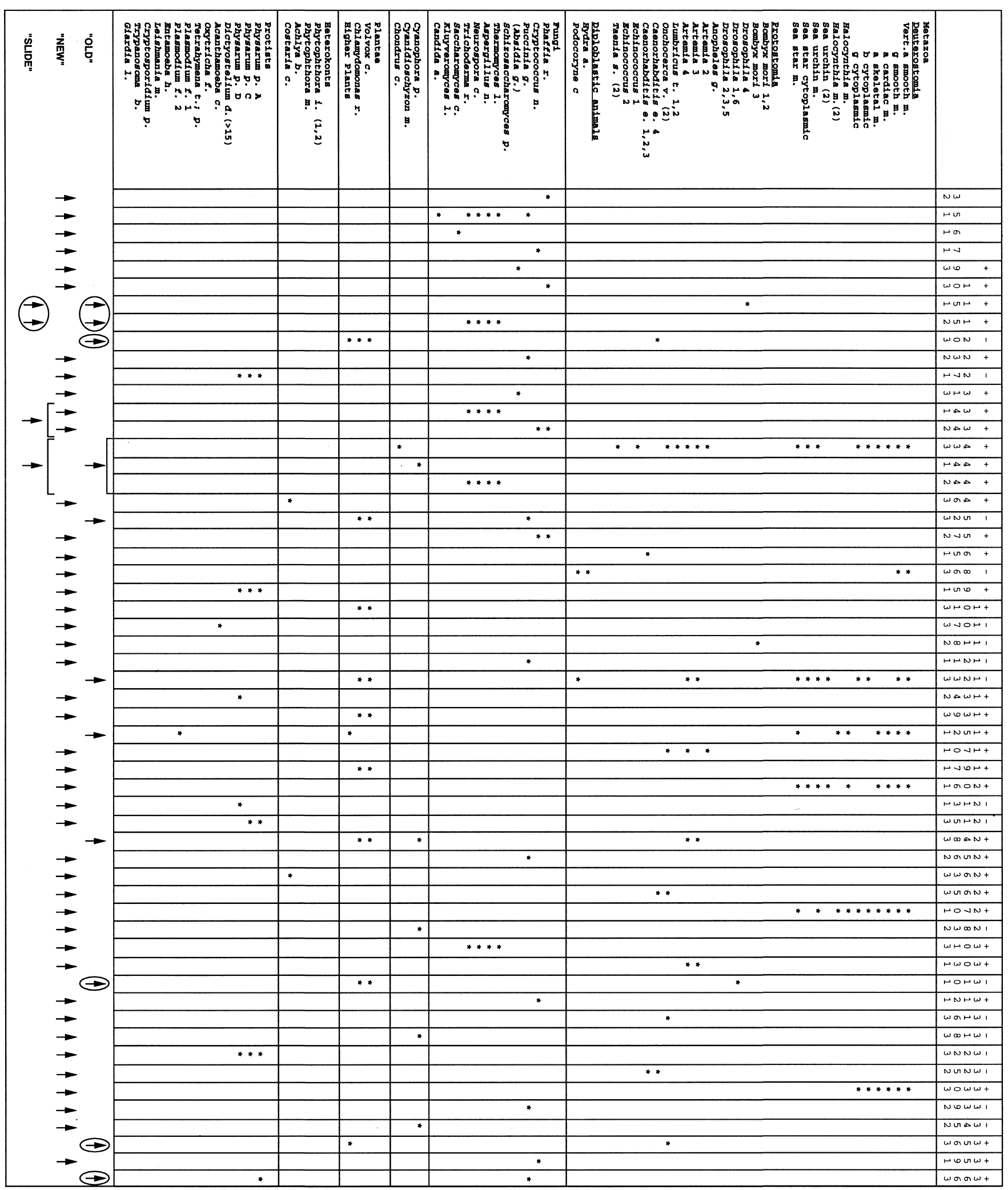

Fig. 4 Catalogue of actin intron positions. Phylogenetic presentation of actin genes available in September 1996 in the EMBL/GenBank databases plus the present study on Cyanophora (U90325). Additional actin genes are from Neurospora c. (D. Gallwitz, personal communication) and Artemia (Ortega et al. 1996). Introns marked by asterisks are characterized by codon- and phase-numbers written vertically at the top. Codon positions are given in reference to the skeletal muscle $\alpha$-actin of vertebrates (for details see Weber and Kabsch 1994). Codons followed by -1 and -2 (last line of heading) are split after the first or second nucleotide, respectively; -3 indicates an intron following the codon. The six distinct genes of vertebrates are from man, mouse, rat, chicken, Xenopus $l$. and carp. The 
283-2, 318-3 and 345-2). Thus there is a random distribution of intron positions with regard to the secondary structural elements of actin (Weber and Kabsch 1994). Using several proteins of known three-dimensional structure and the corresponding gene organisations Stoltzfus et al. (1994) found no significant correspondence between exons and units of protein structure.

\section{Evolutionary analyses of actin introns}

The Cyanophora intron at position 44-1 is separated by only one or two nucleotides from two earlier-established intron positions (Fig. 4). Position 43-3 is relatively common in metazoa and has recently also been found as the sole intron in the actin gene of the red alga Chondrus $c$. (Bouget et al. 1995). An intron at position 44-2 is present in the single-copy actin genes of the fungi Thermomyces $l$., Aspergillus $n$., Neurospora $c$. and Trichoderma $r$. These three closely spaced intron positions (i.e. 43-3, 44-1, and 44-2) could either reflect independent insertions at a hot spot or they could have arisen by sliding of an ancient intron by one, or at most two, nucleotides (see Cerff 1996 for a review of intron sliding). Given the strict length conservation of the actin molecule, except for the very N-terminal end, it does not seem likely that intron sliding is accompanied by the loss or gain of one or more amino acidresidues. However, the minimal mutational events needed to slide an intron by one nucleotide can be a single-nucleotide insertion followed by a compensating deletion, or the reverse order of events. Although the first resulting gene product would be a truncated non-functional actin, the second mutational event could restore a normal actin molecule. Such a mechanism, involving two compensating events, could be tolerated in organisms with several actin

abbreviations ' $m$.' and 'cytoplasmic' stand for muscle and cytoplasmic actins, respectively. Sea urchin genes are from Strongylocentrotus $p$. and Strongylocentrotus f., respectively. Sea star genes are from Pisaster o. Numbers following the species refer to the number(s) of the particular gene(s). Numbers in parenthesis indicate the total number of distinct genes sharing the particular intron pattern. The threeintron pattern of Arabidopsis, soybean, potato, tobacco, rice and maize is referred to as higher plants. The Absidia g. gene lacks information past position 140. An actin gene of Ceratitis c. (mediterranean fruitfly) corresponds to Drosophila genes 1 and 6. Intron positions coinciding with the start or end of a secondary structural element of the actin molecule plus/minus one residue are marked by a plus sign in the top line of the figure. Introns unrelated to such a position are indicated by a minus sign. The first three intron positions are not considered since they occur in the only length-variable region of actin and are found only in some fungi (for details see Weber and Kabsch 1994). Tentative assignments of the "age" of actin introns are shown at the bottom of the figure. In this scheme, "new" introns are lineage- or species-specific, "old" introns are shared between at least two lineages and "slides" are introns that may trace their origin to a single intron that has been translocated 1 or 2 nucleotides from the original site. Circles mark "old" introns that could also be interpreted as representing independent insertional events in two lineages (see text for details) genes and possibly also in organisms with a single actin gene per haploid genome. Two additional hot spots that could also be examples of intron sliding are shown in Fig. 4. Thermomyces and three other fungi with identical actin intron patterns contain an intron at position 34-1 that is one nucleotide removed from the 34-2 intron of the fungi Cryptococcus and Phaffia. The Thermomyces fungal group also contains an intron at position 15-2, one nucleotide removed from the 15-1 intron of the Drosophila actin-4 gene. Since the latter position is unique among the various metazoan genes, including the five other Drosophila genes, we favor the view of an independent insertional event rather than intron sliding.

Based on the intron positions in triose-phosphate isomerase (TIM) genes, Kwiatowski et al. (1995) and Logsdon et al. (1995) argued against the exon theory of genes (Gilbert et al. 1986) and emphasized instead a random insertional origin of introns. Twelve of the twenty one positions within the TIM intron catalogue are thought to be of recent origin. This conclusion is most convincing for intron 5, which is found in only two mosquitos (Culex and Aedes, but not in a further mosquito Anopheles), four other insects, and all other TIM genes analyzed (Kwiatowski et al. 1995; Logsdon et al. 1995). A tentative assignment of actin intron positions in terms of evolutionary origins is indicated in Fig. 4. Introns marked as "new" are lineage(e.g. metazoa, fungi or plants) or species-specific. Introns marked as "old" occur in at least two lineages and may have been present in the common ancestor of crown-group lineages. For none of the "new" introns in the actin catalogue is the assignment of proposed time of origin as clearly delineated as in the case of intron 5 of the TIM catalogue (see above). However actin intron 330-3 is indeed a good candidate for a "new" intron. Found in all vertebrate actin genes it is absent from the sister group of the urochordates (Halocynthia) and other deuterostomia (echinoderms; sea urchins and the sea star) as well as all other known genes. Thus it may be an intron position which was acquired within the vertebrates. The four introns of $\mathrm{Cya}$ nophora that occupy unique positions in the catalogue can be considered as "new" introns. We are aware, however, of the tentative nature of the assignment of a "new" intron in the actin catalogue. In the previous catalogue an intron at position 248-3, for example, seemed specific for the green alga Volvox (Weber and Kabsch 1994; Sheterline et al. 1995). Meanwhile it has not only been found in Chlamydomonas but also in the glaucocystophyte Cyanophora (this study) and in two genes of the arthropod Artemia (Fig. 4). Thus a putatively ,young“ intron can readily turn into an „old“ intron once more genes become known. We also note some uncertainty about the assignment of "old" introns. An intron position which occurs in two lineages, but is only documented by one species, can also reflect two independent insertional events (Fig. 4). Some of these questions may be resolved once more actin genes become available. Alternatively, a more systematic approach to the analysis of actin intron positions in particular lineages or groups of organisms may provide more insights into the origin and loss of introns. 
Acknowledgements D. B. was supported by a grant from the Deutsche Forschungsgemeinschaft (Bh 4/1-2). We thank Wolfgang Berning-Koch for technical assistance and Juergen Ehlting (University of Cologne) for construction of the Cyanophora genomic library.

\section{References}

Baldauf SL, Palmer JD (1993) Animals and fungi are each others closest relatives: congruent evidence from multiple proteins. Proc Natl Acad Sci USA 90:11558-11562

Bhattacharya D, Ehlting J (1995) Actin-coding regions: gene family evolution and use as a phylogenetic marker. Archiv Protistenkd 145:155-164

Bhattacharya D, Medlin L (1995) The phylogeny of plastids: a review based on comparisons of small-subunit ribosomal RNA coding regions. J Phycol 31:489-498

Bhattacharya D, Stickel SK, Sogin ML (1991) Molecular phylogenetic analysis of actin genic regions from Achlya bisexualis (Oomycota) and Costaria costata (Chromophyta). J Mol Evol 33:535-536

Bhattacharya D, Stickel SK, Sogin ML (1993) Isolation and molecular phylogenetic analysis of actin-coding regions from the prymnesiophyte alga, Emiliania huxleyi, using reverse transcriptase and PCR methods. Mol Biol Evol 10:689-703

Bhattacharya D, Helmchen T, Bibeau C, Melkonian M (1995) Comparisons of nuclear-encoded small-subunit ribosomal RNAs reveal the evolutionary position of the Glaucocystophyta. Mol Biol Evol 12:415-420

Bouget F-Y, Kerbourch C, Liaud M-F, de Goër SL, Quatrano RS, Cerff R, Kloareg B (1995) Structural features and phylogeny of the actin gene of Chondrus crispus (Gigartinales, Rhodophyta). Curr Genet 28:164-172

Cavalier-Smith T (1982) The origins of plastids. Biol J Linn Soc 17:289-306

Cavalier-Smith T (1993) Kingdom protozoa and its 18 phyla. Microbiol Rev 57:953-994

Cerff R (1996) The chimeric nature of nuclear genomes and the antiquity of introns as demonstrated by the GAPDH gene system. In: Go M, Schimmel P (eds) Tracing biological evolution in protein and gene structures. Elsevier, Tokyo, pp 205-227

Clark, SW, Meyer DI (1992) Centractin is an actin homologue associated with the centrosome. Nature 359:246-250

Dibb NJ, Newman AJ (1989) Evidence that introns arose at protosplice sites. EMBO J 8:2015-2021

Drouin G, Moniz de Sà M, Zuker M (1995) The Giardia lamblia actin gene and the phylogeny of eukaryotes. J Mol Evol 41:841-849

Felsenstein J (1985) Confidence limits on phylogenies: an approach using the bootstrap. Evolution 39:783-791

Felsenstein J (1993) PHYLIP Manual, Version 3.5. Department of Genetics, University of Washington, Seattle

Gilbert W, Marchionni M, McKnight G (1986) On the antiquity of introns. Cell 46:151-154

Helmchen T, Bhattacharya D, Melkonian M (1995) Analyses of ribosomal RNA sequences from glaucocystophyte cyanelles provide new insights into the evolutionary relationships of plastids. J Mol Evol 41:203-210

Jackson IJ (1991) A reappraisal of non-consensus mRNA splice sites. Nucleic Acids Res 19:3795-3798

Kies L (1979) Zur systematischen Einordnung von Cyanophora paradoxa, Gloeochaete wittrockiana und Glaucocystis nostochinearum. Ber Deutsch Bot Ges Bd 92:445-454

Kies L, Kremer BP (1990) Phylum Glaucocystophyta. In: Margulis L, Corliss JO, Melkonian M, Chapman DJ (eds) Handbook of protoctista. Jones and Bartlett, Boston, pp 152-166

Kimura, M (1980) A simple method for estimating evolutionary rates of base substitution through comparative studies of sequence evolution. J Mol Evol 16:111-120
Kwiatowski J, Krawczyk M, Kornacki M, Bailey K, Ayala FJ (1995) Evidence against the exon theory of genes derived from the triosephosphate isomerase gene. Proc Natl Acad Sci USA 92:85038506

Lees-Miller JP, Helfman DM, Schroer TA (1992) A vertebrate actinrelated protein is a component of a multisubunit complex involved in microtubule-based vesicle motility. Nature 359:244246

Logsdon Jr JM, Tyshenko MG, Dixon C, Jafari JD, Walker VK, Palmer JD (1995) Seven newly discovered intron positions in the triose-phosphate isomerase gene: evidence for the introns-late theory. Proc Natl Acad Sci USA 92:8507-8511

Loomis WF, Smith DW (1990) Molecular phylogeny of Dictyostelium discoideum by protein-sequence comparisons. Proc Natl Acad Sci USA 87:9093-9097

McFadden GI, Gilson PR, Hill DRA (1994) Goniomonas - rRNA sequences indicate that this phagotrophic flagellate is a close relative of the host component of cryptomonads. Eur J Phycol 29:29-32

Melkonian M (1983) Evolution of green algae in relation to endosymbiosis. In: Schenk HEA, Schwemmler W (eds) Endocytobiology II. W. de Gruyter, Berlin, pp 1003-1007

Moniz de Sà M, Drouin G (1996) Phylogeny and substitution rates of angiosperm actin genes. Mol Biol Evol 13:1198-1212

Morden CW, Delwiche CF, Kuhsel M, Palmer JD (1992) Gene phylogenies and the endosymbiotic origin of plastids. BioSystems 28:75-90

Ortega M-A, Díaz-Guerra M, Sastre L (1996) Actin gene structures in two Artemia species, A. franciscana and A. parthenogenetica. J Mol Evol 43:224-235

Palmer JD, Delwiche CF (1996) Second-hand chloroplasts and the case of the disappearing nucleus. Proc Natl Acad Sci USA 93:7432-7435

Query CC, Strobel SA, Sharp PA (1996) Three recognition events at the branch-site adenine. EMBO J 15:1392-1402

Saitou N, Nei M (1987) The neighbor-joining method: a new method for reconstructing phylogenetic trees. Mol Biol Evol 4:406425

Sanger F, Nicklen S, Coulson AR (1977) DNA sequencing with chain-terminating inhibitors. Proc Natl Acad Sci USA 74:54635467

Schlösser UG (1984) Sammlung von Algenkulturen: additions to the collection since 1982. Ber Deutsch Bot Ges 97:465-475

Sheterline P, Clayton J, Sparrow JC (1995) Actin. Prot Profile 2:27-28

Sinibaldi R, Mettler I (1992) Intron splicing and intron-mediated enhanced expression in monocots. Prog Nucleic Acid Res Mol Biol 42:229-257

Sogin ML (1991) The phylogenetic significance of sequence diversity and length variations in eukaryotic small-subunit ribosomal RNA coding regions. Wistar Symp Ser 4:175-188

Sogin ML, Gunderson GJ, Elwood HJ, Alonso RA, Peattie DA (1989) Phylogenetic meaning of the kingom concept: an unusual ribosomal RNA from Giardia lamblia. Science 243:75-77

Stoltzfus A, Spencer DF, Zuker M, Logsdon JM, Doolittle WF (1994) Testing the exon theory of genes: the evidence from protein structure. Science 265:202-207

Swofford DL (1993) PAUP: Phylogenetic analysis using parsimony, V3.1.1. Smithsonian Institution, Washington DC

Swofford DL, Olsen GJ (1990) Phylogeny reconstruction. In: Hillis DM, Moritz C (eds) Molecular systematics. Sinauer, Sunderland, pp 411-501

Wainright PO, Hinkle G, Sogin ML Stickel SK (1993) Monophyletic origins of the Metazoa: an evolutionary link with the fungi. Science 260:340-342

Weber K, Kabsch W (1994) Intron positions in actin genes seem unrelated to the secondary structure of the protein. EMBO J $13: 1280-1286$ 